\title{
Plasma Native Thiol and Lipid Hydroperoxide Levels in the Patients with A Non-Aura Migraine Attack Admitted to the Emergency Department: A Case- Control Study
}

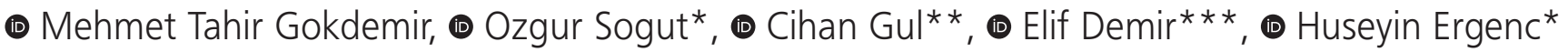 \\ Batman University Faculty of Health, Department of Emergency Medicine, Batman, Turkey \\ * University of Health Sciences Turkey, Haseki Training and Research Hospital, Clinic of Emergency Service, Istanbul, Turkey \\ **Batman University Health Care and Vocational School, Department of Nursing Education, Batman, Turkey \\ ***Harran University Faculty of Medicine, Department of Biochemistry Department, Sanliurfa, Turkey
}

\section{Abstract}

\begin{abstract}
Aim: We emphasize the importance of serum native thiol (SH), lipid peroxidation ( $\mathrm{LOOH})$, and ceruloplasmin (CP) levels in the management of patients with a migraine attack and clarify the possible relationships among these parameters in the emergency department (ED)

Methods: The study was designed as a case-control study which is a type of observational study by including the patients who applied to the University of Health Sciences Turkey, University Diyarbakır Gazi Yasargil Training and Research Hospital between December 2018 and July 2019. A total of 88 participants (study group, $n=46$ and healthy control group, $n=42$ ) consecutively admitted to the ED. with similar demographic characteristics were included in the study. SH, LOOH, and CP levels in both groups were recorded and studied.

Results: The serum SH level was significantly lower in the patient than in the control group $(p=0.002)$. However, the $\mathrm{LOOH}$ and $\mathrm{CP}$ levels were significantly higher in the patient group than in the control group $(p<0.001)$. A significant correlation was observed between $\mathrm{LOOH}$ and $\mathrm{CP}(r=0.332, p=0.002)$. The receiver operating characteristic analysis indicated that a serum $\mathrm{LOOH}$ level of 11.9 $\mu \mathrm{mol} / \mathrm{L}$ predicted an acute migraine attack with $89 \%$ sensitivity and $71 \%$ specificity.
\end{abstract}

Conclusions: Our results suggest that the oxidative status is activated in patients with a non-aura migraine attack, and this may lead to other oxidative and inflammatory processes, with an increase in the serum LOOH level and a decrease in the native thiol level.

Keywords: Non-aura migraine, native thiol, lipid peroxidation, emergency department

\section{Introduction}

Migraine is a neurovascular disease characterized by brain and brain vascular inflammation with episodic attacks. Migraines with and without aura have been described by the International Society of Headaches. There are generally two types of migraine, migraine with aura accompanied by transient focal neurological symptoms that often start with pain, sometimes before headache, and migraine without aura, which is associated with certain symptoms (ICHD) (1). Although many theories on the pathogenesis of migraine have been proposed, it is not yet fully understood. Activation of neuropeptides and mechanisms associated with inflammation are believed to be responsible for the pathogenesis of migraine (2-4).

Thiols are compounds that scavenge free oxygen radicals. Thiols are important antioxidant agents in humans, and thiols containing the sulfur analog in alcohol are in a free or oxidized form in the plasma. Thiol groups are oxidized by disulfide bonds and reduced by reactive oxygen species (5).

Address for Correspondence: Mehmet Tahir Gokdemir, Batman University Faculty of Health,

Department of Emergency Medicine, Batman, Turkey

E-mail: drtahirgokdemir@gmail.com ORCID: orcid.org/ 0000-0002-5546-9653

Received: 10.02.2021 Accepted: 09.05.2021

Copyright 2021 by The Medical Bulletin of istanbul Haseki Training and Research Hospital The Medical Bulletin of Haseki published by Galenos Yayınevi. 
Oxidative stress is responsible for migraine, and the thiol-disulfide balance is likely to deteriorate during a migraine attack. In fact, there are a limited number of studies on migraine patients with a thiol-disulfide balance $(2,5)$. It is clear that these studies are inadequate to explain the clinical and physiopathological status of migraine.

Hydroperoxides ( $\mathrm{LOOH})$, which are more stable than free radicals, in serum, may be markers of oxidative stress in tissue and their levels increase in the presence of stress (5). We hypothesized that acute migraine attacks may be associated with enhanced oxidative status and that determination of the plasma levels of $\mathrm{LOOH}$ and native thiol (NT), which are antioxidants, may be beneficial in the management of patients with migraine in the emergency department (ED). In this study, we emphasize the importance of serum NT and $\mathrm{LOOH}$ levels in the management of patients with a migraine attack and clarify the possible relationships among these parameters. We also investigated the relationships of these parameters with the migraine disability assessment score (MIDAS) and determined Whether NT and $\mathrm{LOOH}$ were independent risk factors in patients with migraine in the ED.

\section{Methods}

\section{Study Design}

The local ethics committee approved the study and all participants gave informed written consent. The study was designed as a case-control study which is a type of observational study by including the patients who applied to the University of Health Sciences Turkey, University Diyarbakır Gazi Yasargil Training and Research Hospital between December 2018 and July 2019 (date: 2018, number: 169). All procedures were carried out in accordance with the ethical standards of the responsible committee on human experimentation and with the Helsinki Declaration. A total of 46 patients with non-aura migraine consecutively admitted to the ED (Patients group: 8 males and 38 females) and 42 healthy controls ( 12 males and 30 females) with similar demographic characteristics were included in this study.

Inclusion criteria: 1 . Admitted to the ED with headache and diagnosed with migraine; $2 \geq 18$ years of age; 3 . any other known chronic or acute disease; and 4 . nonsmoker and no substance dependence.

Exclusion criteria: Patients who were diagnosed with any secondary cause of headache; known febrile illness or other cardiovascular disorder; history of alcohol consumption or alcohol intake; active somatic disease in the past month; active somatic or psychiatric disease; drug use for any reason within 7 days; taking drugs such as alcohol or ecstasy, or taking drugs with suicidal intent; likely to become pregnant or suspected pregnancy; chronic disease; exposed to trauma in the last week; and 12. $<18$ years of age $(n=13)$.

\section{Clinical Evaluation}

The patients were initially treated in the ED. Vital signs such as blood pressure, pulse, and body temperature were evaluated. After a migraine episode was detected, $5 \mathrm{cc}$ of blood were obtained and treatment with non-steroidal anti-inflammatory drugs and/or selective 5HT1B/1D agonists (Triptan) was started. The patients were taken to quiet, low-light observation rooms with appropriate conditions. All of the patients were discharged from the ED after their symptoms were resolved. The age, sex, migraine type, duration of migraine (years), monthly frequency of migraine attacks, duration of migraine attacks (hours), and headache severity [visual analog scale (VAS)] of the patients were recorded. It was not taken into consideration whether the patient received treatment for a migraine attack. Headache severity was evaluated using a VAS (score 1-10). The diagnosis of migraine was made according to the latest diagnostic criteria classification of ICHD (1). Diagnostic criteria of the ICHD for migraine indicate that a patient should have at least five migraine attacks (untreated or unsuccessfully treated) lasting 4-72 hours with at least two of the following characteristics: 1) unilateral placement, 2) a vibrating quality, 3) moderate or severe pain intensity, and 4) aggravation that causes avoidance of daily physical activity. After arriving at the $E D$, the patients were evaluated by the same healthcare professional who used MIDAS to evaluate headacherelated disability. The procedure involved a questionnaire to determine the effect of migraine headaches on the patient's performance routine activity. The patients were divided into three groups according to their MIDAS scores (6).

\section{Laboratory Assessments}

NT and $\mathrm{LOOH}$ levels in both groups were recorded as primary independent variables and demographic and clinical characteristics of the patients and other biochemical and hematological data were recorded as secondary independent variables.

A $5 \mathrm{~mL}$ blood sample was taken within the first hour from all patients who suffered from migraine without aura and from controls. All blood samples were placed in flat tubes and centrifuged at 3,500 rpm for $15 \mathrm{~min}$ to obtain the plasma or serum. All samples were stored in aliquots at $-80{ }^{\circ} \mathrm{C}$ until use.

Hemogram counts were measured using an automated analyzer in K2EDTA samples (Sysmex K-1000; Block Scientific, Bellport, NY USA). Routine biochemical variables such as serum urea, creatinine, sodium, and potassium were measured using commercial kits (Abbott; Block Scientific). Serum CP was measured by the Erel (7) method, an automatic calorimetric method based on enzymatic oxidation of ferrous ion to ferric ion. Details of this method are available in Erel (7). 
Ferrous ion oxidation of xylenol orange was used to assess serum $\mathrm{LOOH}$ levels (8). In the test, the ferric ion concentration was measured using xylenol orange. $\mathrm{LOOH}$ is reduced by triphenylphosphine (TPP), a specific lipid reductant. The presence or absence of TPP during pretreatment determines the difference in $\mathrm{LOOH}$ level. $\mathrm{LOOH}$ values are expressed in $\mu \mathrm{mol} / \mathrm{L}(8)$.

NT concentrations were measured using a new and fully automated analytical method (9). In this method, the reducible disulfide bonds were reduced to form free functional thiol groups. Reductive sodium borohydride was used and extracted with formaldehyde, and the native and reduced thiol groups were determined after reacting with 5.5-dithiobis-(2-nitrobenzoic) acids. Then, the natural thiol levels were measured and calculated. The measurements were performed using a Cobasc 501 instrument (Roche Diagnostics, Mannheim, Germany).

\section{Statistical Analysis}

The demographic and clinical data of the patients were written into excel files at the time of patient admission. Serum biochemical data of the participants were also transferred to this excel file. Study calculations were performed using SPSS version 20.0 software package (Chicago, IL, USA). In the power analysis for SH, differences in the mean levels of patient and control group variables were compared. The power of the test to detect a difference between the two groups was $80 \%$ for NT. The Shapiro-Wilks test was performed to determine the normality of the distribution. Student's t-test was used for comparisons between groups of numerical variables showing a normal distribution, and an analysis of variance (ANOVA)-Tukey test for multiple comparisons within groups. Categorical variables were analyzed using the chi-squared or Fisher's Exact tests. A receiver operating characteristic (ROC) curve analysis was performed to evaluate the discriminatory ability of NT and $\mathrm{LOOH}$. Binary logistic regression analyzes were used for NT and $\mathrm{LOOH}$ in patients with migraine attacks. P-values of $<0.05$ were considered indicative of significance.

\section{Results}

Of the 46 migraine patients included in the study, 8 $(17.4 \%)$ were males, and 38 (82.6\%) were females; $12(28.6 \%)$ of the 42 control individuals were males, and $30(71.4 \%)$ were females. The mean age of the migraine patients was $36.04 \pm 8.06$ years, and that of the control group was $37.32 \pm 12.55$ years. The sex and age distributions were similar between the groups $(p=0.211$, $p=0.509$, respectively). While the baseline clinical characteristics of the patients with acute migraine attacks are shown in Table 1, the demographic and laboratory data of the patient and control groups are in Table 2 .
The serum NT level was meaningfully lower in the patient than in the control group $(0.49 \pm 0.15$ vs. $0.60 \pm 0.19$ $\mu \mathrm{mol} / \mathrm{L}, \mathrm{p}=0.002$, Table 2). However, the LOOH and CP levels were meaningfully higher in the patient group than in the control group (17.81 \pm 5.19 and $407.88 \pm 119.23$ in the patient group; $10.86 \pm 3.05$ and $343.24 \pm 104.83$ in the control group, respectively, $p<0.001, p=0.009$, respectively; Table 2 ).

In Table 3, an ANOVA-Tukey test was performed according to the MIDAS classification to determine the

\begin{tabular}{|c|c|c|}
\hline Pain localization & Number (N) & Proportion (\%) \\
\hline Frontal & 19 & $(41.3 \%)$ \\
\hline Occipital & 7 & $(15.2 \%)$ \\
\hline Temporal & 14 & $(30.4 \%)$ \\
\hline Verteks & 6 & $(13.1 \%)$ \\
\hline Symptoms $\alpha$ & Number (N) & Proportion (\%) \\
\hline Nausea-vomiting & 39 & $(84.8 \%)$ \\
\hline Yawning & 7 & $(15.2 \%)$ \\
\hline Irritability & 15 & $(32.6 \%)$ \\
\hline $\begin{array}{l}\text { Sensitivity to light, sound or } \\
\text { motion }\end{array}$ & 25 & $(54.3 \%)$ \\
\hline Dark circles under the eyes & 4 & $(8.7 \%)$ \\
\hline History & Mean \pm SD & $\begin{array}{l}\text { Minimum- } \\
\text { maximum }\end{array}$ \\
\hline Duration of disease/year & $6.08 \pm 2.42$ & $1.00-12.00$ \\
\hline Attack time/hour & $19.28 \pm 10.43$ & $6.00-48.00$ \\
\hline Frequency of attacks/month & $2.73 \pm 1.52$ & $1.00-7.00$ \\
\hline Pain severity (VAS) & $7.65 \pm 1.40$ & $5.00-10.00$ \\
\hline
\end{tabular}

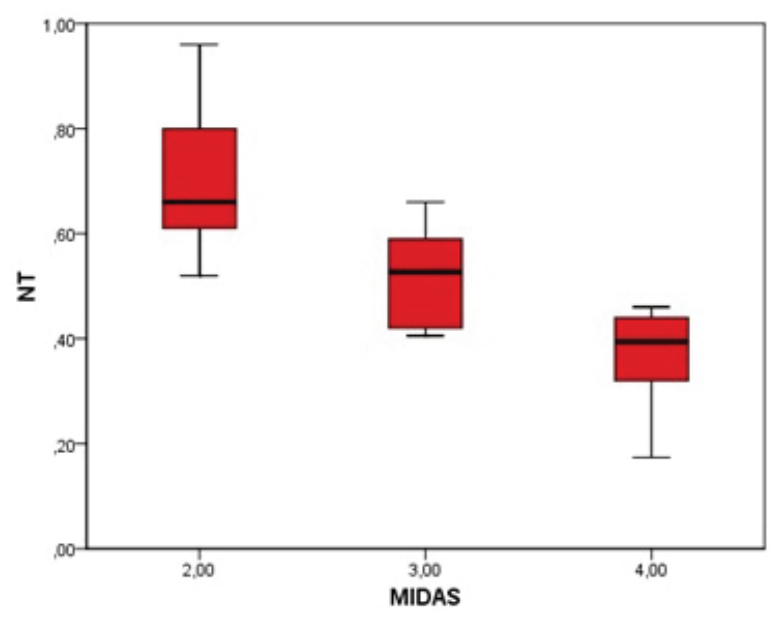

Figure 1. NT level comparison between MIDAS classification in patients with migraine attack

MIDAS: Migraine disability assessment score, NT: Native thiol 
Table 2. Age, gender and baseline laboratory characteristics of patients with acute migraine attack and controls

\begin{tabular}{|c|c|c|c|}
\hline Parameters & $\begin{array}{l}\text { Migraine patients }(52.2 \%, n=46) \\
(n, \% \text { or mean } \pm \text { SD) }\end{array}$ & $\begin{array}{l}\text { Controls }(47.8 \%, n=42) \\
(n, \% \text { or mean } \pm \text { SD) }\end{array}$ & $\mathbf{p}^{*}$ \\
\hline \multicolumn{4}{|l|}{ Gender } \\
\hline (Male/female) & $8 / 38$ & $12 / 30$ & 0.211 \\
\hline Age (years) & $36.04 \pm 8.06$ & $37.32 \pm 12.55$ & 0.509 \\
\hline $\mathrm{LOOH}(\mu \mathrm{mol} / \mathrm{I})$ & $17.81 \pm 5.19$ & $10.86 \pm 3.05$ & $<0.001$ \\
\hline NT (mmol/L) & $0.49 \pm 0.15$ & $0.60 \pm 0.19$ & 0.002 \\
\hline $\mathrm{CP}(\mathrm{mg} / \mathrm{dL})$ & $407.88 \pm 119.23$ & $343.24 \pm 104.83$ & 0.009 \\
\hline LDL cholesterol (mg/dL) & $109.52 \pm 34.81$ & $114.23 \pm 31.06$ & 0.506 \\
\hline $\mathrm{Ca}(\mathrm{mg} / \mathrm{dL})$ & $9.79 \pm 1.66$ & $10.16 \pm 0.92$ & 0.200 \\
\hline Glucose (mg/dL) & $96.10 \pm 24.50$ & $100.98 \pm 23.35$ & 0.313 \\
\hline Urea $(\mathrm{mg} / \mathrm{dL})$ & $23.64 \pm 7.50$ & $29.79 \pm 7.85$ & $<0.001$ \\
\hline Creatinin (mg/dL) & $0.60 \pm 0.16$ & $0.81 \pm 0.19$ & $<0.001$ \\
\hline AST (U/L) & $24.82 \pm 9.70$ & $21.21 \pm 8.25$ & 0.067 \\
\hline $\operatorname{ALT}(U / L)$ & $26.89 \pm 17.52$ & $17.52 \pm 7.34$ & 0.063 \\
\hline T. Chol (mg/dL) & $182.18 \pm 51.14$ & $196.04 \pm 38.15$ & 0.156 \\
\hline $\mathrm{HDL}(\mathrm{mg} / \mathrm{dL})$ & $38.28 \pm 12.61$ & $40.40 \pm 11.99$ & 0.426 \\
\hline $\mathrm{K}(\mathrm{mEq} / \mathrm{L})$ & $4.22 \pm 0.78$ & $4.21 \pm 0.47$ & 0.894 \\
\hline $\mathrm{LDH}(\mathrm{U} / \mathrm{L})$ & $286.75 \pm 101.35$ & $257.18 \pm 94.64$ & 0.162 \\
\hline Hemoglobin $(g / L)$ & $13.93 \pm 4.07$ & $14.20 \pm 1.81$ & 0.715 \\
\hline MPV (fL) & $7.44 \pm 1.27$ & $7.39 \pm 1.08$ & 0.894 \\
\hline PLT (x109/L) & $258.05 \pm 84.76$ & $282.16 \pm 92.47$ & 0.205 \\
\hline APTT (Sec) & $34.14 \pm 4.07$ & $31.95 \pm 7.85$ & 0.258 \\
\hline PT-INR & $1.31 \pm 1.28$ & $1.10 \pm 0.21$ & 0.295 \\
\hline $\mathrm{Hs}-\mathrm{CRP}$ & $0.542 \pm 0.210$ & $0.580 \pm 0.170$ & 0.453 \\
\hline
\end{tabular}

clinical severity of patients presenting to the ED with a migraine attack. The mean NT level of the group with the most severe clinical presentation was lowest (for comparisons among three groups, $p<0.001$; Table 3 and Figure 1, respectively). In contrast, the mean plasma $\mathrm{LOOH}$ level increased in proportion to the clinical severity of migraine ( $p=0.002$, Table 3, Figure 2$)$.

A significant, positive correlation was observed between MIDAS score and LOOH ( $r=0.449, p=0.002)$. Conversely, the MIDAS score was significantly negatively correlated with NT level $(r=-0.777, p<0.001)$. As seen in Figure 3, the ROC analysis indicated that a cut-off serum $\mathrm{LOOH}$ level of $11.9 \mu \mathrm{mol} / \mathrm{L}$ predicted acute a migraine attack with $89 \%$ sensitivity and $71 \%$ specificity (area under the curve $=0.896$; $95 \%$ confidence interval $(\mathrm{Cl})$ : 0.832-0.960).

In binary logistic regression analyses, NT and $\mathrm{LOOH}$ were independent markers of a migraine attack in the ED (Table 4); the odds ratio (OR) was significantly lower for NT (OR<0.001; 95\% Cl, 0.00-1.04; $\mathrm{p}=0.002$ ) and significantly higher for LOOH (OR: 1.89; 95\% Cl, 1.42-2.52; $\mathrm{p}<0.001$ )

\section{Discussion}

This is one of the few studies in which serum NT was studied in patients who presented to the ED with a migraine attack without aura. No previous study has evaluated NT and LOOH levels in patients admitted to the ED with a migraine attack without aura. Our results show that the NT level was meaningfully lower and the $\mathrm{LOOH}$ level was higher in patients admitted to the ED with a migraine attack without aura compared to those of healthy participants. Moreover, the NT level decreased and the $\mathrm{LOOH}$ level increased in proportion to the severity of the migraine attack. Also, the MIDAS score was significantly positively correlated with the $\mathrm{LOOH}$ level and significantly negatively correlated with that of NT. In addition, binary logistic regression analyses showed that the NT and $\mathrm{LOOH}$ levels were independent markers of a migraine attack in the ED.

Migraine patients are susceptible to neurovascular reactions caused by different factors. The Impaired balance between inhibition and stimulation of the nervous system leads to some clinical variations, including headache 


\begin{tabular}{|l|l|l|l|l|l|}
\hline \multicolumn{6}{|l|}{ Table 3. Serum NT, CP and LOOH levels comparison between MIDAS classification in patients with migraine attack } \\
\hline MIDAS & N & Mean \pm SD & Minimum-maximum & ANOVA & Tukey HSD \\
\hline Clas & \multicolumn{3}{|l|}{} & Px & P $\alpha$ \\
\hline NT II & 8 & $0.70 \pm 0.14$ & $0.52-0.96$ & $<0.001$ & II \& III $\rightarrow<0.001$ \\
\hline III & 20 & $0.51 \pm 0.08$ & $0.41-0.66$ & - & II \& IV $\rightarrow<0.001$ \\
\hline IV & 18 & $0.36 \pm 0.09$ & $0.17-0.46$ & - & III \& IV $\rightarrow<0.001$ \\
\hline LOOH II & 8 & $13.75 \pm 2.55$ & $10.78-17.30$ & 0.002 & II \& III $\rightarrow 0.112$ \\
\hline III & 20 & $17.58 \pm 3.37$ & $10.63-24.10$ & - & II \& IV $\rightarrow 0.005$ \\
\hline IV & 18 & $20.04 \pm 5.91$ & $12.10-32.52$ & - & III \& IV $\rightarrow 0.218$ \\
\hline CP II & 8 & $456.09 \pm 110.25$ & $297.86-647.50$ & 0.426 & II \& III $\rightarrow 0.436$ \\
\hline III & 20 & $393.92 \pm 119.33$ & $244.20-641.84$ & - & II \& IV $\rightarrow 0.541$ \\
\hline IV & 18 & $401.95 \pm 123.89$ & $268.26-678.03$ & - & III \& IV $\rightarrow 0.977$ \\
\hline $\begin{array}{l}\text { P } \alpha: \text { Tukey test for Multiple Comparisons (the mean difference is significant at the 0.05 level), Px: One-Way ANOVA test. MIDAS: Migraine disability assessment score, LB: } \\
\text { Lower bound, UB: Upper bound, CP: Ceruloplasmin, NT: Native thiol, LOOH: Lipid peroxidation }\end{array}$ \\
\hline
\end{tabular}

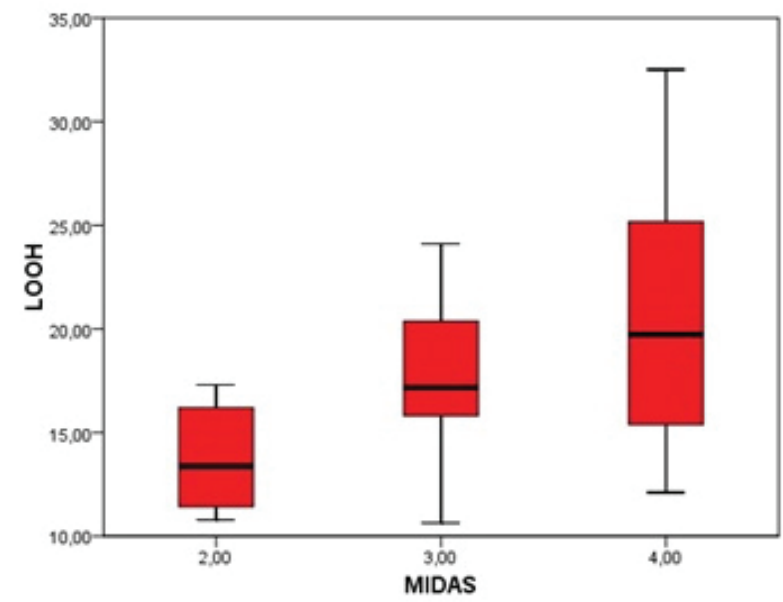

Figure 2. LOOH level comparison between MIDAS classification in patients with migraine attack

LOOH: Lipid peroxidation, MIDAS: Migraine disability assessment score

$(10,11)$. Clinical studies have indicated that oxidative stress increases and antioxidant defense decreases in patients with migraine $(12,13)$. The role of the acute inflammatory response caused by oxidative stress has been reported in some clinical studies in patients with a headache due to a migraine $(13,14)$. $\mathrm{LOOH}$ is a primary product of lipid peroxidation, and it may be useful in predicting oxidative stress in the blood (15). Few studies have shown levels of lipid peroxidation in migraine patients or the levels of these antioxidant enzymes $(12,16)$.

Lipid peroxidation levels can be assessed based on serum total oxidative stress measures using a recently developed method $(16,17)$. Native thiol may be useful in determining antioxidant activity. Gumusyayla reported that NT levels were higher in migraine patients than in healthy controls (5). In the same study, NT level was reported to be similar in migraine patients with and without aura. In
Receiver operating characteristic curve for $\mathrm{LOOH}$

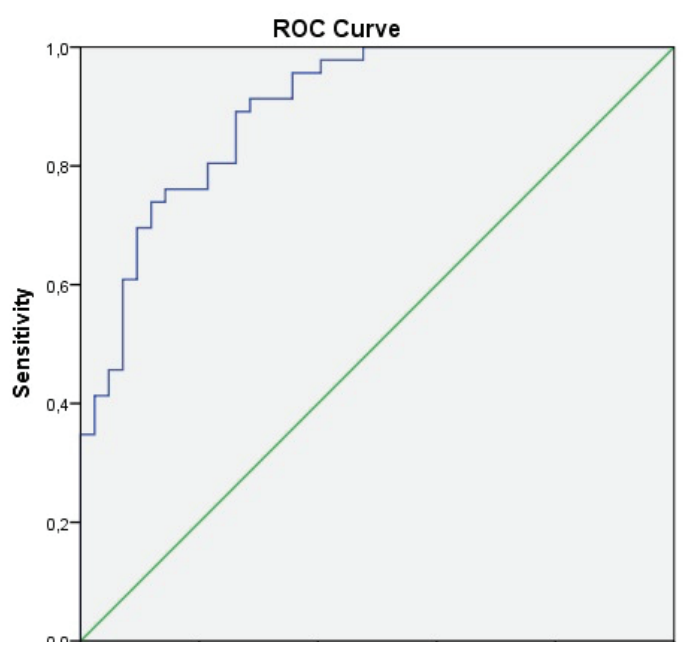

Figure 3. Receiver operating characteristic analysis for NT NT: Native thiol, LOOH: Lipid peroxidation, $\mathrm{LOOH}$ : Lipid peroxidation ROC: Receiver operating characteristic

\begin{tabular}{|l|l|l|l|l|l|}
\hline \multicolumn{6}{|c|}{ Table 4. Binary logistic regression analyses of study variables } \\
\hline Variables & $\begin{array}{l}\text { OR } \\
\mathbf{9 5 \%}\end{array}$ & Cl & $\mathbf{p}$ & Sensitivity & Specificity \\
\hline NT & $<0.001$ & $0.00-1.04$ & 0.002 & & \\
\hline LOOH & 1.89 & $1.42-2.52$ & $<0.001$ & 84.3 & 85.7 \\
\hline CP & 1.00 & $0.99-1.01$ & 0.317 & & \\
\hline Hs-CRP & 0.48 & $0.18-1.29$ & 0.149 & & \\
\hline $\begin{array}{l}\text { NT: Native thiol, LOOH: Lipid peroxidation, CP: Ceruloplasmin, Hs-CRP: High- } \\
\text { sensitivity-C-reactive protein, OR: Odds ratio, Cl: Confidence interval }\end{array}$ \\
\hline
\end{tabular}

another study, the relationships of changes in dynamic thiol-disulfide homeostasis with pain severity and duration of disease were investigated in children with migraine (14). In that study, a negative correlation was found between the NT level and headache severity in patients with both 
migraine and tension-type headaches. NT levels have been reported to decrease as headache severity increases. In our study, serum NT levels were meaningfully lower in patients who presented to the ED with a migraine attack without aura than in the control group. We also classified the patients with migraine according to their MIDAS score; the mean NT level was significantly lower in patients with the most severe migraine attacks. Moreover, there was a negative correlation between the MIDAS score and NT level.

In a study of patients with coronary slow flow, the $\mathrm{LOOH}$ levels were higher than those in the normal population (18). Similarly, Yigit et al. (13) reported that the levels of oxidative parameters were high in patients presenting to the ED with migraine attacks. Similarly, levels of $\mathrm{CP}$ and $\mathrm{LOOH}$, which are inflammatory markers closely associated with oxidative stress, were meaningfully higher in migraine patients than in controls in the present study. Furthermore, the level of $\mathrm{LOOH}$, the main product of lipid peroxidation and a marker of the level of oxidative stress, increased meaningfully. In addition, the mean plasma level of $\mathrm{LOOH}$ increased in proportion to the severity of the migraine attack according to the MIDAS classification; patients with the highest MIDAS score had the highest level of $\mathrm{LOOH}$. So, there was a significant, positive correlation between the MIDAS score and the LOOH level. However, the patients in the MIDAS-II, -III, and -IV groups had similar CP levels. Moreover, the NT and LOOH levels were independent markers of a migraine attack in the ED. The high serum $\mathrm{LOOH}$ levels suggest that oxidative stress plays a role in migraine. The oxidant state is dominant in cases of an acute attack. The increased serum levels of $\mathrm{LOOH}$ during migraine attack suggest that an acute inflammation event is triggered in the dominant state of oxidation. However, whether oxidative stress is a cause of migraine or the result of metabolic processes associated with this condition is unclear.

\section{Study Limitations}

The limitations of our study should be mentioned. The migraine diagnosis was based on subjective criteria. This was a single-center study with a relatively small number of participants. We used a pain severity scale for migraine patients, and we could not evaluate the effects of previously used drugs that may have affected the levels of $\mathrm{SH}, \mathrm{LOOH}$, or $\mathrm{CP}$. This study was based on a single blood sample to determine NT, $\mathrm{LOOH}$, and $\mathrm{CP}$ levels in each patient. A comparison could not be made regarding the pain location and symptoms of the patients, because the primary parameters in our study are based on the analysis of laboratory data. The fact that such a comparison was not made in the study is an important limiting factor. In our study, we can say that dehydration may affect oxidative stress. In this respect, perhaps this is an important limitation of our study.

\section{Conclusion}

The levels of the antioxidant NT were meaningfully lower in patients with migraine without aura than in controls. Moreover, as the MIDAS score increased, the level of NT decreased and the severity of disability increased. In contrast, the $\mathrm{LOOH}$ level, which reflects the oxidative state, was meaningfully higher in migraine patients than in the controls. Moreover, we observed a positive correlation between MIDAS score and LOOH level. In addition, NT and $\mathrm{LOOH}$ levels were independent risk factors for migraine attack in the ED. Our results suggest that the oxidative status is activated in patients with a migraine, and this may lead to other oxidative and inflammatory processes, with an increase in the serum $\mathrm{LOOH}$ level and a decrease in the NT level.

\section{Authorship Contributions}

Concept: M.T.G., Design: O.S., Data Collection or Processing: C.G., E.D., Analysis or Interpretation: O.S., M.T.G., Literature Search: H.E., Writing: O.S., M.T.G.

Conflict of Interest: No conflict of interest was declared by the authors.

Financial Disclosure: The authors declared that this study received no financial support.

\section{References}

1. Göbel CH, Karstedt SC, Münte TF, et al. ICHD-3 is significantly more specific than ICHD-3 beta for diagnosis of migraine with aura and with typical aura. J Headache Pain 2020;21:2.

2. Albay VB, Tütüncu M. Evaluation of the Relationship Between Thyroid Dysfunction and Menstrual Migraine in Adult Females. Med Bull Haseki 2020;58:110-4.

3. Poyrazoğlu HG, Öztürk AB. Predictive value of laboratory parameters in childhood migraine. Acta Neurol Belg 2020;120:907-14.

4. Gross EC, Putananickal N, Orsini AL, et al. Mitochondrial function and oxidative stress markers in higher-frequency episodic migraine. Sci Rep 2021;11:4543.

5. Gumusyayla S, Vural G, Bektas H, Neselioglu S, Deniz O, Erel O. A novel oxidative stress marker in migraine patients: dynamic thiol-disulphide homeostasis. Neurol Sci 2016;37:1311-7.

6. Dönmez H ,Yoldaş TK, Solak EB, Çolpak Al. Demographic/ Clinical Characteristics and Midas Scores of 103 Consecutive Migraine Patients. Turk J Neurol 2008;14:388-93.

7. Erel O. Automated measurement of serum ferroxidase activity. Clin Chem 1998;44:2313-9.

8. Nourooz-Zadeh J. Ferrous ion oxidation in presence of xylenol orange for detection of lipid hydroperoxides in plasma. Methods Enzymol 1999;300:58-62. 
9. Erel O, Neselioglu S. A novel and automated assay for thiol/ disulphide homeostasis. Clin Biochem 2014;47:326-32.

10. Yu ES, Priyadharsini S S Y, Venkatesan T. Migraine, Cyclic Vomiting Syndrome, and Other Gastrointestinal Disorders. Curr Treat Options Gastroenterol 2018;16:511-27.

11. Söğüt Ö, Kaya H, Gökdemir MT, et al. Early Oxidative Status in Adult Patients With İsolated Traumatic Brain Injury. Turk J Med Sci 2012;42:1010-9.

12. Kim S, Bae DW, Park SG, Park JW. The impact of Pain-related emotions on migraine. Sci Rep 2021;11:577.

13. Yigit $M$, Sogut $O$, Tataroglu Ö, et al. Oxidative/antioxidative status, lymphocyte DNA damage, and urotensin-2 receptor level in patients with migraine attacks. Neuropsychiatr Dis Treat 2018;14:367-74.

14. Eren Y, Dirik E, Neşelioğlu S, Erel Ö. Oxidative stress and decreased thiol level in patients with migraine: cross-sectional study. Acta Neurol Belg 2015;115:643-9.
15. Argüelles S, Gómez A, Machado A, Ayala A. A preliminary analysis of within-subject variation in human serum oxidative stress parameters as a function of time. Rejuvenation Res 2007; 10:621-36.

16. Bolayir E, Celik K, Kugu N, Yilmaz A, Topaktas S, Bakir S. Intraerythrocyte antioxidant enzyme activities in migraine and tension-type headaches. J Chin Med Assoc 2004;67:2637.

17. Papurica M, Rogobete AF, Sandesc $D$, et al. Redox Changes Induced by General Anesthesia in Critically III Patients with Multiple Traumas. Mol Biol Int 2015;2015:238586.

18. Baysal SS, Koc S. Oxidant-Antioxidant balance in patients with coronary slow flow. Pak J Med Sci 2019;35:786-792. 